\title{
Associação entre força muscular periférica e força muscular respiratória em idosos hospitalizados
}

\section{Association between peripheral muscle force and muscular respiratory strength in hospitalized elderly}

\section{Patricia Maia Botelho', Laís França Rios², Marília de Souza Maia, Mychelle Regina Melo de Souza Luz ${ }^{4}$, Mario Cezar Macedo Silva Junior ${ }^{5}$, Sérgio Luis Figueiredo de Jesus ${ }^{6}$, Jorge Luis Motta dos Anjos ${ }^{7}$, Bruno Prata Martinez ${ }^{8}$}

${ }_{1}^{1}$ Autora para correspondência. Universidade do Estado da Bahia. Salvador, Bahia, Brasil. ORCID: 0000-0001-9491-2374. patriciam.botelho@outlook.com 2Universidade do Estado da Bahia. Salvador, Bahia, Brasil. ORCID: 0000-0001-9654-7382. Ifrios@uneb.br 3Universidade do Estado da Bahia. Salvador, Bahia, Brasil. ORCID: 0000-0002-4510-5821. mariliasmaia.fisio@gmail.com ${ }^{4}$ Universidade do Estado da Bahia. Salvador, Bahia, Brasil. ORCID: 0000-0002-4547-1944. mychellefisiouneb@gmail.com 5Universidade do Estado da Bahia. Salvador, Bahia, Brasil. ORCID: 0000-0003-4220-1313. cezarmacedojunior@gmail.com 6Universidade do Estado da Bahia. Salvador, Bahia, Brasil. ORCID: 0000-0001-5978-3351. sergiosIfj@gmail.com ${ }^{7}$ Centro Universitário Maurício de Nassau. Hospital Geral Roberto Santos. Salvador, Bahia, Brasil. ORCID: 0000-0003-2897-9858. jorgelmanjos@hotmail.com ${ }^{8}$ Universidade Federal da Bahia. Universidade do Estado da Bahia. Salvador, Bahia, Brasil. ORCID: 0000-0002-4673-8698. brunopmartinez@hotmail.com

\begin{abstract}
RESUMO | INTRODUÇÃO: A sarcopenia é uma síndrome caracterizada por perda progressiva e generalizada de massa e força do músculo esquelética, bem como possivelmente da musculatura respiratória com risco de desfechos adversos, como declínio da funcionalidade, má qualidade de vida e morte. O risco de complicações respiratórias e infecções na população idosa é elevado, o que pode ser devido, em parte, as alterações da força do diafragma. OBJETIVO: Avaliar a associação entre força muscular periférica e força muscular respiratória em idosos hospitalizados. MÉTODOS: Trata-se de um estudo realizado em um hospital público na cidade de Salvador, Bahia. As variáveis primárias mensuradas foram medidas antropométricas, força de preensão palmar, força muscular respiratória através da aferição da pressão inspiratória máxima (PImáx), velocidade de marcha e presença de tabagismo. As variáveis secundárias extraídas a partir de dados de prontuário foram idade, gênero, diagnóstico médico admissional, perfil clínico admissional (clínico ou cirúrgico), tempo de internação no momento da coleta e índice de comorbidades de Charlson. Para avaliação entre as variáveis da FPP e força muscular respiratória foi utilizado a análise da correlação de Pearson. RESULTADOS: Dos 95 idosos avaliados, 73,7\% era do sexo masculino, $89,5 \%$ tinham internação por motivo cirúrgico $(40,2 \%$ cirurgias abdominais; $30,4 \%$ cirurgias urológicas; $16,3 \%$ amputações; $13,1 \%$ outras cirurgias), IMC $24,5 \pm 4,1 \mathrm{Kg} / \mathrm{m} 2$. A média de idade foi $68,1 \pm 6,1$ anos, força de preensão palmar $31,3 \pm 9,1 \mathrm{Kgf}$ e PImáx $-77,5 \pm 33,2 \mathrm{~cm}$ H2O. O tempo para avaliação inicial foi de 4,3 $\pm 3,1$ dias após internação hospitalar. $O$ índice de comorbidades de Charlson foi de $3,6 \pm 1,89$ e a pontuação do mini-exame do estado mental foi $22,9 \pm 7,22$. A correlação entre a força de preensão palmar e a força muscular respiratória foi moderada ( $R=-$ $0,439$ e valor de $p=0,001)$. CONCLUSÃo: Existe relação entre FMR e FMP, bem como a FMR pode predizer dinapenia. Apesar disso, recomenda-se que a avaliação da FMR e FMP sejam feitas de forma individualizada para um posterior direcionamento do plano terapêutico, já que esta relação foi apenas moderada.
\end{abstract}

ABSTRACT I INTRODUCTION: Sarcopenia is a syndrome characterized by the progressive and widespread loss of skeletal muscle mass and strength, which can be used as a life-threatening respiratory massage, such as decline in function, poor quality of life, and death. The risk of respiratory and control complications in the elderly population is one that may be due to a change in the strength of the diaphragm. OBJECTIVE: To assess the association between peripheral muscle strength and respiratory muscle strength in hospitalized elderly. METHODS: this is a study carried out in a public hospital in Salvador, Bahia. The primary variables measured were anthropometric measurements, palmar grip strength, respiratory muscle strength through maximal inspiratory pressure (MIP), gait velocity and presence of smoking. The secondary variables extracted from medical records were age, gender, admission medical diagnosis, admission clinical profile (clinical or surgical), length of hospital stay at time of collection and Charlson comorbidities index. The Pearson correlation analysis was used to evaluate the PPF variables and respiratory muscle strength. RESULTS: Of the 95 elderly patients evaluated, $73.7 \%$ were male, $89.5 \%$ were hospitalized for surgical reasons $(40.2 \%$ were abdominal surgeries, $30.4 \%$ were urological surgeries, $16.3 \%$ were amputations, $13.1 \%$ were other surgeries ), BMI $24.5 \pm 4.1 \mathrm{~kg} / \mathrm{m} 2$. The mean age was $68.1 \pm 6.1$ years, palmar grip strength $31.3 \pm 9.1 \mathrm{Kgf}$ and PImax $-77.5 \pm 33.2 \mathrm{~cm} \mathrm{H} 2 \mathrm{O}$. The time for initial evaluation was $4.3 \pm 3.1$ days after hospital admission. The Charlson comorbidities index was $3.6 \pm 1.89$ and the mini-mental status score was $22.9 \pm 7.22$. The correlation between palmar grip strength and respiratory muscle strength was moderate $(R=-0.439$ and $p=0.001)$. CONCLUSION: There is a relationship between FMR and FMP, and FMR can predict dynapenia. Despite this, it is recommended that the evaluation of FMR and FMP be made in an individualized way for a subsequent direction of the therapeutic plan, since this relationship was only moderate.

KEYWORDS: Breathing. Elderly. Muscle strength. 


\section{Introdução}

A sarcopenia é uma síndrome caracterizada por perda progressiva e generalizada de massa e força do músculo esquelético, com risco de desfechos adversos, como declínio da funcionalidade, má qualidade de vida e morte ${ }^{1}$. De acordo com o Grupo de Trabalho Europeu sobre Sarcopenia em Pessoas Idosas (EWGSOP), recomenda-se usar baixa massa muscular e baixa função muscular (força muscular ou desempenho físico) como critérios para definir o diagnóstico de sarcopenia. O estadiamento pode orientar sobre o manejo clínico dessa condição e pode ser classificada como pré-sarcopenia, sarcopenia ou sarcopenia grave ${ }^{3}$.

Já a dinapenia pode ser definida como a perda de força muscular associado à idade que não é causada por doenças neurológicas ou musculares, predispondo idosos a um risco aumentado de limitações funcionais e mortalidade 5 . Os fatores que contribuem para a dinapenia são provavelmente multifatoriais e incluem aspectos dos sistemas nervoso e muscular. Dados Iongitudinais recentes sugerem que a perda progressiva de força muscular é de 2-5 vezes maior que a perda de massa muscular com o envelhecimento ${ }^{6}$.

Em relação à prevalência de sarcopenia, seguindo os critérios do EWGSOP, em idosos da comunidade, esse percentual foi de 1 a $29 \%$, conforme dados de recente revisão sistemática ${ }^{7}$. Já em pacientes idosos hospitalizados, a taxa relatada variou entre $21,8 \%$ a $26 \% 9$. Há uma lacuna na literatura quanto à prevalência desse percentual em relação à dinapenia, justificada pela falta de consenso na definição padrão do termo. Apenas um estudo trouxe que a condição mais prevalente na população idosa é a dinapenia, quando comparado a sarcopenia e sarcodinapenia, sendo a taxa de $34,4 \%$ em mulheres e $25,8 \%$ em homens ${ }^{10}$.

Recentemente evidenciou-se que o envelhecimento está associado também à perda de força do músculo diafragma, reduzindo a capacidade ventilatória e de força ${ }^{11}$. As alterações musculares associadas à idade afetam a função muscular respiratória, apresentando redução significativa de $25 \%$ na força do diafragma de idosos quando comparado a adultos jovens ${ }^{12-13}$. Por isso, o desenvolvimento de complicações respiratórias é comum ${ }^{14}$, sendo a causa mais prevalente de morte nessa população ${ }^{15}$. A redução de força do diafragma é devido a atrofia seletiva das fibras musculares do tipo Ilx e/ou Ilb, enquanto as fibras do tipo I e lla são preservadas ${ }^{16}$. Estes indivíduos apresentam aumento da proteólise da fibra elástica (elastina) e aumento do colágeno no parênquima pulmonar, bem como aumento da rigidez da caixa torácica, favorecendo uma desvantagem mecânica dos músculos respiratórios e podem, em longo prazo, gerar fraqueza na musculatura respiratória ${ }^{17}$. Como a dinapenia ainda é um assunto recente na literatura científica e sabendo da escassez de estudos demonstrando a associação entre força muscular periférica e força muscular respiratória em idosos hospitalizados, o presente estudo teve como objetivo avaliar à correlação entre força muscular periférica e força muscular respiratória, bem como avaliar a acurácia da fraqueza muscular respiratória para predizer dinapenia em idosos hospitalizados.

\section{Métodos}

\section{Desenho do estudo e população}

Trata-se de um estudo observacional sobre associação da força muscular respiratória e força muscular periférica em idosos hospitalizados, realizado em um hospital público referência localizado na cidade de Salvador, Bahia, no período de outubro de 2017 a setembro de 2018.

Foram incluídos idosos com idade igual ou superior a 60 anos, que apresentavam índice de massa corporal (IMC) $<30$ kg/m2; período de internação entre o primeiro e o quinto dia de hospitalização; capacidade de compreensão e execução de comandos externos simples, como: levantar a perna, abrir os olhos e outros comandos verbais simples, ausência de uso de drogas vasoativas e/ou inotrópicas e capacidade de deambulação sem uso de órteses ou auxilio do fisioterapeuta (conforme liberação expressa em prontuário por parte dos médicos assistentes).

Os idosos que apresentavam dor, dispneia, alteração cardiorrespiratória incapacitante ou doenças respiratórias prévias foram excluídos do estudo. Além disso, o estudo excluiu idosos que durante a realização dos testes apresentaram precordialgia, saturação periférica de oxigênio (SpO2) $\leq 90 \%$, tontura, palidez, náuseas, sudorese, palpitações, pré-síncope, dispneia, perda de dispositivos invasivos ou queda.

Os participantes foram recrutados após aprovação no Comitê de Ética, sob o protocolo CAAE 54038316.0.0000.5028, respeitando todos os princípios 
éticos e a resolução 466/12. Uma avaliação diária dos prontuários foi realizada com o objetivo de selecionar indivíduos internados na unidade hospitalar e que estivessem dentro dos critérios de inclusão propostos pelo estudo ao longo do período.

\section{Variáveis primárias e secundárias}

As variáveis primárias mensuradas neste estudo foram medidas antropométricas (peso corporal e altura), mini-exame do estado mental (MEEM), força de preensão palmar e força muscular respiratória. Já as variáveis secundárias foram extraídas a partir de dados de prontuário, incluindo idade, gênero, diagnóstico médico admissional, perfil admissional (clínico ou cirúrgico), tempo de internação no momento da coleta e índice de comorbidades de Charlson.

O IMC foi obtido através da divisão do peso corporal em quilogramas pela altura ao quadrado em metros. Os valores encontrados foram classificados de acordo com os dados da Organização Mundial da Saúde: baixo peso (IMC <18,5), eutrofia (IMC entre 18,5-24,99), sobrepeso (IMC entre 25-29,99) e obesidade (IMC $\geq 30,00) 18$. A função cognitiva foi avaliada através do mini-exame do estado mental (MEEM), que quantifica as diversas funções cognitivas, com um escore que varia entre 0 a 30 pontos $^{19}$.

A medida de força muscular periférica foi realizada através do dinamômetro manual Saehan (Saehan Corporation, 973, Yangdeok-Dong, Masan 630-728, Korea). Os participantes foram instruídos a sentar-se em uma cadeira, com cotovelos a $90^{\circ}$ e realizarem uma força máxima no dinamômetro de preensão palmar manual ${ }^{20}$. Três medidas foram realizadas com um intervalo de um minuto entre elas, sendo considerada para análises posteriores, a maior medida. $\mathrm{O}$ critério para definição de fraqueza muscular e/ou dinapenia foi força de preensão palmar inferior a $20 \mathrm{kgf}$ nas mulheres e $30 \mathrm{kgf}$ nos homens ${ }^{21}$.

A medida da força muscular inspiratória direta foi realizada através de um manuvacuômetro Wika $\mathrm{CL}$ 1.6. Os indivíduos foram instruídos a sentar-se com quadril em flexão a $90^{\circ}$ e pés apoiados no chão e orientados sobre a realização das técnicas. O manuvacuômetro foi acoplado ao ramo expiratório da válvula unidirecional e a mesma acoplado ao bocal ${ }^{22}$. Foi solicitada uma expiração lenta e completa até o volume residual e, em seguida, uma inspiração profunda até a capacidade pulmonar total e logo após uma expiração máxima até o volume residual, sob os comandos do avaliador. A medida obtida foi o maior valor encontrado de três medidas, com variação menor que 5 pontos e um intervalo entre elas de $1 \mathrm{mi}$ nuto, recomendado pela American Thoracic Society ${ }^{23}$.

Para quantificar o nível de gravidade das doenças, foi coletado o índice de comorbidades de Charlson nas primeiras 24 horas da admissão. O índice de Charlson é um escore composto por vinte condições clínicas selecionadas empiricamente com base no efeito sobre o prognóstico de uma coorte de pacientes internados num serviço de medicina geral dos Estados Unidos ${ }^{24}$. A sua pontuação varia de 0 a 6 , para algumas condições clínicas, sendo que a cada década, a partir dos 50 anos, acrescenta-se um peso ao índice. Quanto maior a pontuação obtida, maior a gravidade e risco de óbito.

\section{Análise estatística}

Os resultados foram descritos em médias e desviopadrão para as variáveis numéricas e em percentuais para as variáveis categóricas. Para avaliação da correlação entre as variáveis força de pensão palmar e força muscular respiratória foi utilizado a análise de correlação de Pearson. Para avaliação da acurácia da força muscular respiratória para predizer dinapenia foi feito a descrição da curva ROC (Receiver Operator Caracteristics) e sua respectiva acurácia, sendo considerado significante um valor de $p<0,05$. A análise estatística e o banco de dados foi realizada no programa SPSS, v10.0 (Chicago, Illinois, USA).

\section{Resultados}

A amostra foi composta por noventa e cinco idosos hospitalizados, com média de idade de $68,13 \pm 6,05$ anos, tendo predomínio do sexo masculino $(73,7 \%)$ e o perfil admissional prevalente cirúrgico (89,5\%). Os motivos mais prevalentes nessa amostra foram pós-operatório de cirurgia abdominal $(38,9 \%)$ e pósoperatório de cirurgia urológica $(29,5 \%)$, seguido de pós operatório para amputações $(15,8 \%)$, pós operatório cardíaco $(1,1 \%)$, doenças gastrointestinais $(7,4 \%)$, doença vascular $(1,1 \%)$, doença do trato urinário $(3,2 \%)$ e outros $(3,2 \%)$. Os valores médios de força muscular periférica e força muscular respiratória encontrados nesse estudo foram de 31,3 $\pm 9,1 \mathrm{kgf}$ e $-77,6 \pm 33,33 \mathrm{~cm} \mathrm{H20}$ ), respectivamente. Os dados descritivos da amostra estão contidos na Tabela 1. 


\begin{tabular}{lll}
\hline & Média / DP & N / \% \\
\hline Idade, anos & $68,13 \pm 6,05$ & \\
IMC, kg/m $\mathbf{m}^{2}$ & $24,51 \pm 4,08$ & $70(73,7)$ \\
Gênero & & $25(26,3)$ \\
Masculino & & $9(9,5)$ \\
Feminino & $4,37 \pm 3,17$ & $85(89,5)$ \\
Tempo internação durante a coleta, dias & & $1(1,1)$ \\
Perfil admissional & & \\
Clínico & & \\
Cirúrgico & & \\
Omissos & & \\
Índíce Charlson & $3,62 \pm 1,89$ & \\
MEEM & $22,90 \pm 1,80$ & \\
FPP & $31,34 \pm 9,09$ & \\
PiMáx & $-77,55 \pm 33,20$ & \\
\hline FPP: força de preensão palmar; PIMáx: pressão inspiratória máxima & \\
PO: pós-operatón
\end{tabular}

FPP: força de preensão palmar; PIMáx: pressão inspiratória máxima PO: pós-operatório.

Houve moderada correlação entre força muscular periférica e força muscular respiratória $(R=-0,439$; valor de $p=0,001)$ na figura 1 .

Figura 1. Correlação entre a força muscular periférica e força muscular respiratória na amostra de idosos hospitalizados.(n=95)

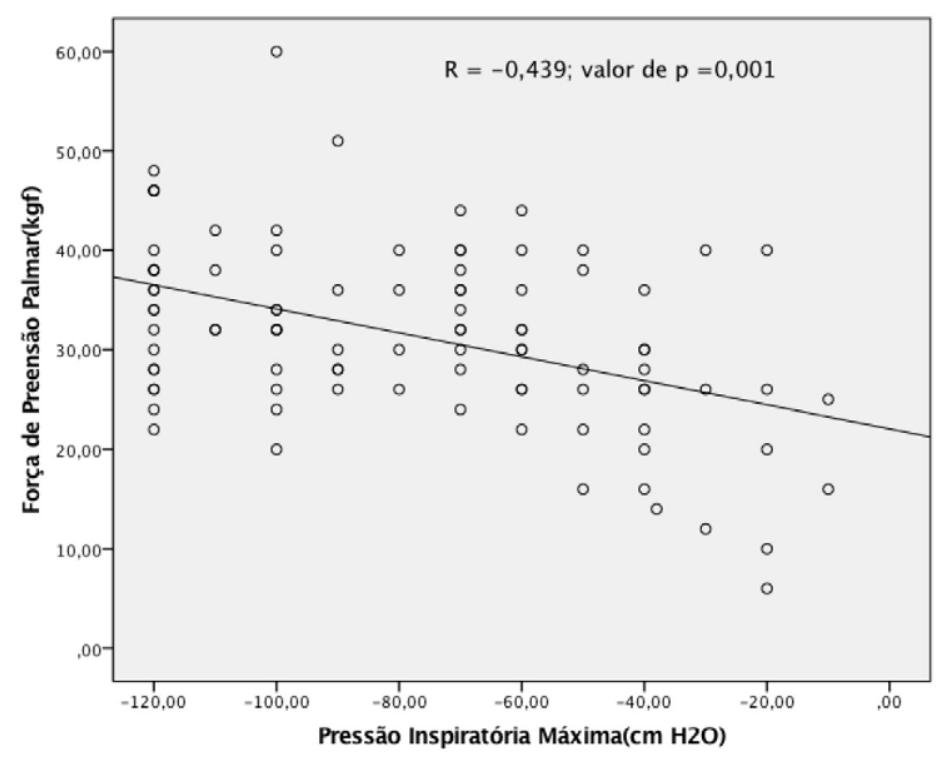

$\mathrm{Na}$ análise da capacidade da força muscular respiratória predizer dinapenia também foi observada moderada acurácia (acurácia=0,66; IC 95\%=0,54-0,79; p=0,011) na figura 2. 


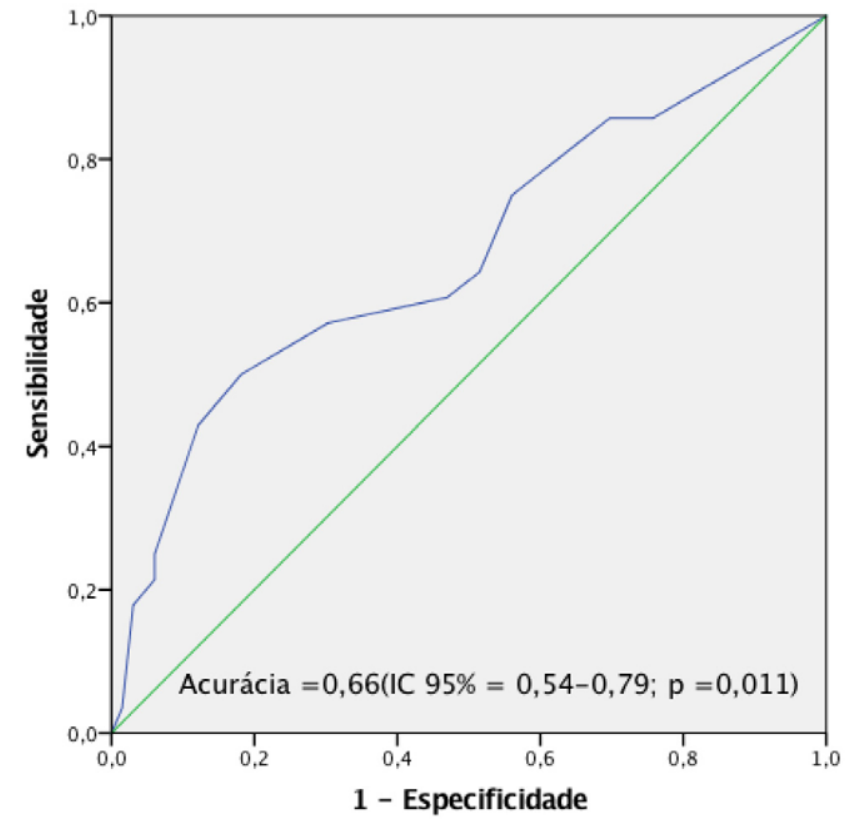

\section{Discussão}

Os resultados desse estudo mostraram que há moderada correlação entre força muscular periférica e força muscular respiratória em idosos hospitalizados, além de acurácia moderada na capacidade da força muscular respiratória predizer dinapenia, o que reforça uma possível associação entre o músculo respiratório e periférico.

Os dados do presente estudo estão em concordância com outros estudos $25-27,34$, os quais identificaram que a força muscular periférica também teve correlação moderada e positiva com a força muscular inspiratória. Os autores enfatizam, ainda, que a sarcopenia pode causar diminuição do pico de fluxo de tosse, trazendo possíveis complicações respiratórias com o envelhecimento ${ }^{25,26}$. Esses estudos sugerem que existe relação significativa entre a força muscular respiratória, especialmente os músculos inspiratórios, e a força muscular dos membros e a massa muscular em idosos.

A correlação entre força de preensão manual e pressão máxima inspiratória (Pimáx) parece ser forte em indivíduos saudáveis $(r=0,76)^{28}$, e a Pimáx pode ser prevista com precisão de $80 \%$ por parâmetros importantes, como o sexo, IMC e força de preensão manual. Além disso, também houve correlação positiva entre a força muscular inspiratória (Pimáx) com a força muscular periférica e com índices sarcopênicos em adultos jovens saudáveis ${ }^{29}$, enquanto a cor- relação no presente estudo foi moderada $(r=0,439)$. Porém, esses resultados podem ser conflitantes com este estudo, já que a população incluída era de idosos hospitalizados, sem qualquer doença pulmonar prévia e sem limitações funcionais importantes.

Além disso, a força de preensão palmar também foi correlacionada positivamente com a função pulmonar de maneira dose-dependente ${ }^{30}$ em idosos sem doença pulmonar prévia ou doenças crônicas. Os autores trazem que a detecção oportuna de força de preensão palmar reduzida pode ser útil na avaliação de possível comprometimento na função pulmonar.

Como a sarcopenia é um processo generalizado, acredita-se que também haja comprometimento dos músculos respiratórios, o que possivelmente resulte em ventilação prejudicada, redução de mobilidade e perda de independência funcional. Recentemente, a sarcopenia do músculo diafragma em ratos idosos foi demonstrada ${ }^{31}$. Os autores avaliaram camundongos jovens (5 meses), idosos (23 meses) e "BubR1 h/h (envelhecimento acelerado) e observaram que o envelhecimento está associado a perda de força no músculo diafragma, representada pelas reduções na força e no tamanho da fibra muscular tipo I ou lla. A presença de sarcopenia muscular respiratória é provavelmente um fator central para o desenvolvimento de insuficiência respiratória e redução de depuração das vias aéreas entre os indivíduos mais velhos. 
A sarcopenia do músculo diafragma (DIAm) ainda não foi bem caracterizada. O DIAm é o principal músculo inspiratório e o conhecimento da sarcopenia DIAm é importante para estabelecer os efeitos do envelhecimento na função respiratória. A pressão transdiafragmática máxima medida durante um esforço é aproximadamente $25 \%$ menor em idosos saudáveis quando comparado a jovens do sexo masculino ${ }^{12}$, sugerindo que as alterações musculares relacionadas ao envelhecimento podem predispor a fadiga muscular durante esforços respiratórios extenuantes.

Nesse estudo, houve moderada acurácia na capacidade da força muscular respiratória predizer dinapenia. A redução de força muscular respiratória em idosos sem doença pulmonar prévia está associada, ainda, a perda de mobilidade, independente da força muscular periférica e nível de atividade física ${ }^{32}$. Autores identificaram ainda, que a redução de força muscular respiratória está associada a pior desempenho físico nos idosos, independentemente da massa e força muscular periférica ${ }^{33}$. Sugere-se, portanto, que os dados de pressão inspiratória máxima (PiMáx) podem ser úteis para fins prognósticos na população idosa.

O pico de fluxo expiratório (PEF) é determinado pela força dos músculos respiratórios. Porém, pesquisas sobre o uso do PEF como medida de sarcopenia são limitadas, não sendo recomendado como medida de força muscular respiratória isolado ${ }^{3}$. Os estudos relacionados à força muscular respiratória e sarcopenia utilizaram como medida a pressão máxima inspiratória (PiMax), sendo que os valores médios de referência em indivíduos idosos saudáveis encontrados foram de $57 \mathrm{cmH} 20$ para mulheres e $83 \mathrm{cmH} 20$ para homens. O valor de PiMáx encontrado no presente estudo foi $77,55 \pm 33,20$ sendo semelhante ao resultado de outros estudos ${ }^{34}$. Os valores descritos na literatura são escassos, porém autores identificaram um ponto de corte para PiMax de moderada precisão, em idosos do sexo masculino, de $-55,6 \mathrm{cmH} 20^{34}$. Recentemente, um estudo trouxe como pontos de corte da pressão máxima inspiratória entre $\leq-55 \mathrm{cmH} 20$ e $\leq-45 \mathrm{cmH} 20$ para homens e mulheres, respectivamente ${ }^{35}$. Os autores mostraram ainda que esses valores estão associados ao diagnóstico de sarcopenia em idosos.

Dessa forma, percebemos que a redução de força muscular periférica e respiratória pode acarretar em aumento dos custos econômicos de saúde, como maior tempo de internação hospitalar e mortalidade, além de custos de reabilitação e a necessidade de cuidados institucionais na alta. Esse estudo teve algumas limitações como o fato de indivíduos dessa amostra não apresentarem fraqueza muscular respiratória e periférica e esses dados podem reduzir a validade externa para outras populações, como idosos que apresentem redução de força. Estudos futuros são necessários para avaliar as variáveis de força por um tempo maior e com um maior número de indivíduos idosos.

\section{Conclusão}

Existe relação entre FMR e FMP, bem como a FMR pode predizer dinapenia. Apesar disso, recomenda-se que a avaliação da FMR e FMP sejam feitas de forma individualizada para um posterior direcionamento do plano terapêutico, já que esta relação foi apenas moderada. A prevenção da sarcopenia no ambiente hospitalar, com programas de reabilitação focados em treinamento de força e resistência da musculatura periférica e respiratória, podem ser vistos com importância para prevenção ou manutenção da força e massa muscular em pessoas idosas.

\section{Contribuições dos autores}

Anjos JLM, Luz MRMS e Martinez BP participaram da concepção e desenho do estudo. Botelho PM, Rios LF, Maia MS, Luz MRMS, Silva Junior MCM, Jesus SLF, Anjos JLM e Martinez BP participaram da coleta e análise dos dados da pesquisa, interpretação dos dados e redação do artigo científico.

\section{Conflitos de interesses}

Nenhum conflito financeiro, legal ou político envolvendo terceiros (governo, empresas e fundações privadas, etc.) foi declarado para nenhum aspecto do trabalho submetido (incluindo, mas não se limitando a subvenções e financiamentos, participação em conselho consultivo, desenho de estudo, preparação de manuscrito, análise estatística, etc.).

\section{Referências}

1. Delmonico MJ, Harris TB, Lee JS, Visser M, Nevitt M, Kritchevsky $\mathrm{SB}$ et al. Alternative definitions of sarcopenia, lower extremity performance, and functional impairment with aging in older men and women. J Am Geriatr Soc. 2007;55(5):769-74. doi: 10.1111/j.1532-5415.2007.01140.x 
2. Cruz-Jentoft AJ, Bahat G, Bauer J, Boirie Y, Bruyère O, Cederholm T et al. Sarcopenia: European consensus on definition and diagnosis. Age and Ageing 2010;48(1):16-31. doi: 10.1093/ageing/afy169

3. Delmonico MJ, Harris TB, Visser M, Park SW, Conroy MB, VelasquezMieyer $\mathrm{P}$ et al. Longitudinal study of muscle strength, quality, and adipose tissue infiltration. Am J Clin Nutr. 2009;90(6):1579-1585. doi: 10.3945/ajcn.2009.28047

4. Cruz-Jentoft A, Landi F, Schneider S, Zúñiga $C$, Arai $H$, Boirie $Y$ et al. (2014). Prevalence of and interventions for sarcopenia in ageing adults: a systematic review. Report of the International Sarcopenia Initiative (EWGSOP and IWGS). Age Ageing. 2014;43(6):748-59. doi: 10.1093/ageing/afu115

5. Martinez B, Batista AK, Gomes IB, Olivieri FM, Camelier FW, Camelier AA. Frequency of sarcopenia and associated factors among hospitalized elderly patients. BMC Musculoskelet Disord. 2015;4(4):745-5. doi: 10.1186/s12891-015-0570-x

6. Alexandre TS, Duarte YA, Santos JL, Lebrão ML. Prevalence and associated factors of sarcopenia, dynapenia, and sarcodynapenia in community-dwelling elderly in São Paulo - SABE Study. Rev Bras Epidemiol. 2019;21(Suppl 2):e180009. doi: 10.1590/1980549720180009.supl.2

7. Tolep K, Higgins N, Muza S, Griner G, Kelsen SG. Comparison of diaphragm strength between healthy adult elderly and young men. Am J Respir Crit Care Med. 1995;152(2):677-82. doi: 10.1164/ ajrccm.152.2.7633725

8. Heron M. Deaths: leading causes for 2011. Nati Vital Stat Rep. 2015;64(7):1-96.

9. Elliott JE, Greising SM, Mantilla CB, Sieck GC. Functional impact of sarcopenia in respiratory muscles. Respir Physiol Neurobiol. 2016;226:137-46. doi: 10.1016/j.resp.2015.10.001

10. Lalley PM. The aging respiratory system-Pulmonary structure, function and neural control. Respir Physiol Neurobiol. 2013;187(3):199-210. doi: 10.1016/j.resp.2013.03.012

11. Reis MM, Arantes PMM. Medida da força de preensão manual - validade e confiabilidade do dinamômetro Saehan. Fisioterapia e Pesquisa. 2011;18(2):176-81. doi: 10.1590/S180929502011000200013

12. Alexandre TS, Duarte YAO, Santos JLF, Wong R, Lebrão ML. Sarcopenia according to the European Working Group on Sarcopenia in Older People (EWGSOP) versus dynapenia as a risk factor for disability in the elderly. J Nutr Health Aging. 2014;18(5):547-53. doi: 10.1007/s12603-013-0424-x

13. American Thoracic of Spirometry. "Standardization of Spirometry, 1994 Update. American Thoracic Society. Am J Respir Crit Care Med. 1995;152(3):1107-36. doi: 10.1164/ ajrccm.152.3.7663792
14. Charlson ME, Pompei P, Ales KL, MacKenzie CR. A new method of classifying prognostic comorbidity in longitudinal studies: development and validation. J Chronic Dis 1987;40(5):373-83. doi: 10.1016/0021-9681(87)90171-8

15. Bahat G, Tufan A, Ozkaya H, Tufan F, Akpinar TS, Akin S et al. Relation between hand grip strength, respiratory muscle strength and spirometric measures in male nursing home residents. Aging Male. 2014;17(3):136-40. doi: 10.3109/13685538.2014.936001

16. Simoes LA, Dias JMD, Marinho KC, Pinto CLLR, Britto RR. Relação da função muscular respiratória e de membros inferiores de idosos comunitários com a capacidade funcional avaliada por teste de caminhada. Rev Bras Fisiot. 2010;14(1): doi: 10.1590/S1413$\underline{35552010000100005}$

17. Shin HI, Kim DK, Seo KM, Kang SH, Lee SY, Son S. Relation Between Respiratory Muscle Strength and Skeletal Muscle Mass and Hand Grip Strength in the Healthy Elderly. Ann Rehabil Med. 2017;41(4):686-692. doi: 10.5535/arm.2017.41.4.686

18. Efstathiou ID, Mavrou IP, Grigoriadis KE. Correlation Between Maximum Inspiratory Pressure and Hand-Grip Force in Healthy Young and Middle-Age Individuals. Respir Care. 2016;61(7):925-9. doi: $10.4187 /$ respcare.04319

19. Ro HJ, Kim DK, Lee SY, Seo KM, Kang SH, Suh HC. Relationship Between Respiratory Muscle Strength and Conventional Sarcopenic Indices in Young Adults: A Preliminary Study. Ann Rehabil Med. 2015;39(6):880-887.

20. Son DH, Yoo JW, Cho MR, Lee YJ. Relationship Between Handgrip Strength and Pulmonary Function in Apparently Healthy Older Women. J Am Geriatr Soc. 2018;66(7):1367-1371. doi: 10.1111/ jgs.15410

21. Greising SM, Mantilla CB, Gorman BA, Ermilov LG, Sieck GC. Diaphragm muscle sarcopenia in aging mice. Exp Gerontol. 2013;48(9):881-7. doi: 10.1016/j.exger.2013.06.001

22. Buchman AS, Boyle PA, Wilson RS, Leurgans S, Shah RC, Bennett DA. Respiratory muscle strength predicts decline in mobility in older persons. Neuroepidemiology. 2008;31(3):174-180. doi: $\underline{10.1159 / 000154930}$

23 Giua R, Pedone C, Scarlata S, Carrozzo I, Rossi F, Valiani V et al. Relationship Between Respiratory Muscle Strength and Physical Performance in Elderly Hospitalized Patients. Rejuvenation Research. 2014;17(4):366-71. doi: 10.1089/rej.2014.1549

24. Izawa K, Watanabe S. Respiratory muscle strength in relation to sarcopenia in elderly cardiac patients. Aging Clin Exp Res. 2016;28(6):1143-1148. doi: 10.1007/s40520-016-0534-5

25. Ohara DG, Pegorari MS, Oliveira SNL, Silva FR, Monteiro RL, Matos AP et al. Respiratory Muscle Strength as a Discriminator of Sarcopenia in Community-Dwelling Elderly: A Cross-Sectional Study. J Nutr Health Aging. 2018;22(8):952-958. doi: 10.1007/ s12603-018-1079-4 